\title{
A un año de la pandemia COVID 19: ¿Cómo están los recursos hídricos?
}

\section{One year after the pandemic COVID 19: How are the water resources?}

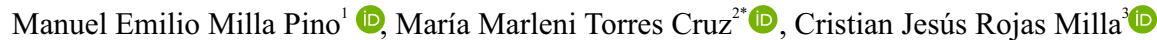

\section{RESUMEN}

El presente trabajo tiene como finalidad analizar el estado actual de los recursos hídricos habiendo transcurrido un año desde que se decretó el estado de emergencia generado por la pandemia COVID 19, desarrollando los siguientes aspectos, recursos hídricos antes de la pandemia, medidas implementadas durante la pandemia, metodología de valoración de impactos ambientales e impactos de las medidas implementadas durante la pandemia en los recursos hídricos. Se tomó como base la metodología expuesta por Conesa (1997), de la cual se seleccionaron cinco criterios, específicamente, naturaleza, intensidad, extensión, momento y sinergia, y estos fueron aplicados a las cinco medidas de mayor restricción implementadas durante la pandemia COVID 19, obteniéndose como resultados que la medida con mayor puntaje es el cierre temporal de fronteras con $100 \mathrm{y}$ la medida con menor puntaje es llevar mascarilla con 38,75. Mientras que el puntaje total es 356,25, que se traduce en una moderada incidencia en los recursos hídricos.

Palabras claves: recursos hídricos, estado de emergencia, COVID 19.

\begin{abstract}
The purpose of this work is to analyze the current state of water resources, a year having elapsed since the state of emergency generated by the COVID 19 pandemic was decreed, developing the following aspects, water resources before the pandemic, measures implemented during the pandemic, methodology for assessing environmental impacts and impacts of the measures implemented during the pandemic on water resources. The methodology set out by Conesa (1997) was taken as a basis, from which five criteria were selected, specifically, nature, intensity, extension, moment and synergy, and these were applied to the five most restrictive measures implemented during the COVID 19 pandemic, obtaining as results that the measure with the highest score is the temporary closure of borders with 100 and the measure with the lowest score is wearing a mask with 38.75 . While the total score is 356.25 , which translates into a moderate impact on water resources.
\end{abstract}

Keywords: water resources, state of emergency, COVID 19.

\footnotetext{
Facultad de Ingeniería Civil, Universidad Nacional de Jaén, Jaén, Perú. ${ }^{2}$ Facultad de Ingeniería Forestal y Ambiental, Universidad Nacional de Jaén, Jaén, Perú. ${ }^{3}$ Facultad de Ciencias Básicas, Universidad del Atlántico, Barranquilla, Colombia.

"Autor de correspondencia. E-mail: marle.torres@unj.edu.pe
} 


\section{INTRODUCCIÓN}

Los recursos hídricos antes de la implementación de las medidas de aislamiento social obligatorio como estrategia para controlar la pandemia COVID 19, sufrían las consecuencias de un accionar desproporcionado y hay veces hasta irracional, por parte de la población, lo cual se evidenciaba por los altos niveles de contaminación que presentaban los cuerpos y corrientes de aguas, trayendo como consecuencias limitantes para el desarrollo de los seres vivos acuáticos así como también en el agua de consumo humano. Las acciones de confinamiento impuestas a la sociedad han restringido las labores y actividades que regularmente se venían realizando, tales como, visita a espacios acuáticos con fines de esparcimiento, turismo y recreación, aspecto que ha incidido en la disminución del volumen de desechos contaminantes y en la depuración de los reservorios de agua, generando condiciones favorables para la masificación progresiva de las especies vegetales y/o animales que se desarrollan en ambientes hídricos.

Al respecto, es conveniente señalar que, según las estadísticas de la última década, indican que la demanda mundial de agua se ha estimado en alrededor de $4.600 \mathrm{~km} 3 /$ año y se prevé que aumente entre un $20 \%$ y un $30 \%$ (de 5.500 a 6.000 km3/año) para 2050 (Burek, 2016; WWAP, 2018; 2019; 2020; 2021). Por otro lado, el uso global del agua ha aumentado seis veces en los últimos 100 años (Wada, 2016) y sigue creciendo de manera constante a una tasa aproximada del $1 \%$ anual (AQUASTAT, 2021).

En cuanto al Perú, es preciso señalar que es el octavo país con mayor disponibilidad hídrica y concentra el $1,9 \%$ del agua dulce en el mundo. Sin embargo, enfrenta un déficit hídrico en la vertiente del Pacífico. Las fuentes de agua superficial vienen disminuyendo en los últimos 50 años. Se proyecta que los recursos hídricos disminuyan entre 5\% y $10 \%$ en los próximos 10 años (WIDENZA CONSULTORES, 2021).

Este trabajo, tiene como propósito fundamental contextualizar, analizar y discutir el efecto que han tenido hasta la actualidad, las medidas implementadas por el estado para controlar los embates de la pandemia COVID 19 en la calidad de los recursos hídricos.

\section{MATERIAL Y MÉTODOS}

\section{Recursos hídricos antes de la pandemia}

El agua dulce es un recurso limitado y su calidad está bajo presión constante. Preservar la calidad del agua dulce es importante para el abastecimiento de agua potable, la producción de alimentos y el uso de aguas recreativas (WWAP, 2018; 2019; 2020; 2021).

Antes de la pandemia, la dinámica social estaba orientada hacia lo que podemos calificar como un uso irracional de los recursos hídricos, lo cual ponía en riesgo la disponibilidad y calidad de agua potable, así como para las actividades de extracción y cultivo marino, tanto costeras como continentales, producción agropecuaria y conservación del ambiente acuático, y en consecuencia, atentaba contra el normal abastecimiento de ese vital líquido. Esto indica que la cantidad de agua disponible responde a la conciencia de la población y en este orden de ideas Hunter et al. (2010) señalan que la cantidad de agua a la que se puede acceder está determinada por condiciones socio-culturales. En lo referente a la calidad de agua se ha observado antes de la pandemia altos niveles de contaminación, generados como consecuencia del accionar irresponsable de la población, la cual vierte sus desechos líquidos y sólidos a las corrientes y cuerpos de aguas sin medir el impacto negativo que esto trae en los recursos hídricos, como lo es la disminución progresiva de agua de calidad disponible para consumo humano.

En este sentido, Ki-moon (2014) expresó que la calidad de cualquier masa de agua, superficial o subterránea depende tanto de factores naturales como de la acción humana. El deterioro de la calidad del agua se ha convertido en motivo de preocupación a nivel mundial con el crecimiento de la población humana, la expansión de la actividad industrial y agrícola y la amenaza del cambio climático como causa de importantes alteraciones en el ciclo hidrológico.

En la mayoría de los países se observa escasez de agua de calidad, aspecto que influye no solo en la salud 
humana, sino también en la producción agrícola, pecuaria e industrial, ya que la inversión en mecanismos tendientes a lograr la depuración del recurso hídrico es alta, y, por consiguiente, tiene un fuerte impacto en la sustentabilidad y sostenibilidad económica de los procesos productivos, trayendo como consecuencia un decrecimiento del volumen de producción.

Los problemas de calidad del agua persisten tanto en los países desarrollados como en los países en desarro1lo, e incluyen la pérdida de cuerpos de agua de calidad óptima, los impactos asociados con los cambios en la hidromorfología, el aumento de contaminantes emergentes y la propagación de especies invasoras (WWAP, 2018).

El déficit de agua de calidad en comunidades de bajos recursos atenta sustancialmente contra sus posibilidades de desarrollo, ya que los procesos contaminantes antrópicos siguen en franco crecimiento y niegan la oportunidad de mejora de la calidad de vida. Es por ello que WWAP (2019), indica que la calidad del agua es una preocupación creciente en las áreas rurales, tanto en países de ingresos bajos como altos. En muchos países, hoy en día la mayor fuente de contaminación del agua es la agricultura, mientras que, en todo el mundo, el contaminante químico más común encontrado en los acuíferos subterráneos es el nitrato derivado de la agricultura.

En el Perú, se cuenta una sólida disponibilidad de recursos hídricos, que permite satisfacer los requerimientos de la población, tanto para consumo humano como para uso productivo, pero como consecuencia del crecimiento demográfico y expansión territorial, la mayor parte del recurso hídrico se consume en las ciudades, generando una desproporcionada distribución en el ámbito nacional.

Al respecto, el Informe técnico N. ${ }^{\circ}$ 004-2014-ANA DGCRH, concluye que los recursos hídricos del país presentan diferencias significativas en cuanto a su disponibilidad debido al crecimiento demográfico, el cambio de uso territorial que prioriza el uso urbano, la desigual distribución espacial del agua y su variabili- dad estacional.

El Perú posee una vasta riqueza natural mineralógica por las condiciones geológicas que inciden en la calidad de los recursos hídricos. Sumado a ello, las acciones transformadoras que ejerce el hombre sobre el ambiente pueden ocasionar impactos positivos o negativos, que alteraran la calidad de los cuerpos naturales de agua continentales y marino-costeros, debido a las actividades productivas y poblacionales. En una primera aproximación de la situación de los recursos hídricos, se recopiló información de calidad de los recursos hídricos durante los años 2000-2012 y se logró establecer una base con un total de 64030 datos procedentes de 331 monitoreos en 59 unidades hidrográficas.

Los resultados de la calidad de los recursos hídricos del periodo 2000-2012 determinan que los parámetros coliformes termotolerantes, arsénico, plomo y cadmio (II) asociados a las descargas de aguas residuales poblacionales, pasivos ambientales mineros, minería informal (donde corresponden) y características naturales por la geología muestran niveles de afectación a la calidad de los recursos hídricos con fines de riego (en las tres vertientes) y para fines poblacionales (vertientes del Pacífico y Amazonas). Además, los parámetros coliformes termotolerantes, DBO5 y plomo (vertiente del Amazonas); DBO5, cadmio, arsénico y plomo (vertiente del Pacífico) y pH, arsénico, cadmio, plomo y mercurio (vertiente del Titicaca), muestran niveles de afectación a la calidad de los recursos hídricos con fines de conservación del ambiente acuático. Estos se encuentran asociados a las descargas de aguas residuales poblacionales, pasivos ambientales mineros, minería informal (donde corresponden) y otros vinculados a las características naturales de las cuencas hidrográficas.

Como resultado de los monitoreos realizados hasta el año 2014, la ANA ha identificado 41 unidades hidrográficas cuyos parámetros exceden los ECA-Agua20083. Se evidencia que la principal causa de afectación de los cuerpos de agua del país es el vertimiento de aguas residuales domésticas y municipales, que 
modifican o alteran la calidad del agua con bacterias fecales, materia orgánica, amoniaco, nitritos y otros parámetros que no permiten su utilización para los usos agrícola, producción de agua potable y conservación del ambiente acuático, entre otros.

Durante el periodo 2013-2014, se registró un total de 4147 fuentes de afectación en las tres vertientes hidrográficas. La vertiente del Pacífico registró 2283 fuentes, que corresponde al 55\%; la vertiente del Amazonas 1602 (39\%), y la vertiente del Titicaca con 262 fuentes $(6 \%)$. Siendo, de manera similar al anterior, las descargas de aguas residuales poblacionales la fuente de mayor presión sobre los recursos evaluados (1709 fuentes, $41 \%$ respecto al total), seguido de la disposición inadecuada de los residuos sólidos (911 fuentes y $22 \%$ del total).

De acuerdo a la Estrategia Nacional para el Mejoramiento de la Calidad de los Recursos Hídricos, el ANA (2016) identificó 41 unidades hidrográficas cuyos parámetros de calidad exceden los ECA-Agua, siendo la causa principal el vertimiento de aguas residuales industriales, domésticas y municipales.

El Diagnóstico de la Calidad de los Recursos Hídricos del Perú, correspondiente a un periodo de evaluación iniciado desde abril de 2010 a diciembre de 2012, señala que de un total de 159 unidades hidrográficas, 35 de ellas presentaron, en promedio, concentraciones de los parámetros $\mathrm{pH}$, conductividad eléctrica, coliformes termotolerantes, demanda bioquímica de oxígeno, arsénico, mercurio, cadmio, plomo y hierro, por encima de los ECA-Agua aprobados el año 2008 (correspondientes a la Clasificación de los cuerpos naturales de agua superficial aprobada con Resolución Jefatural $N^{o}$ 202-2010-ANA). Este resultado está asociado a los vertimientos de aguas residuales no autorizados, pasivos ambientales, residuos sólidos y condiciones naturales (factores geológicos, ambientales e hidrológicos).

\section{Medidas implementadas durante la pandemia}

La OMS en 2020, indica como medidas preventivas de la COVID-19, que la comunidad debe adoptar algunas medidas de precaución, tales como, mantener el dis- tanciamiento físico, llevar mascarilla, ventilar bien las habitaciones, evitar las aglomeraciones, lavarse las manos y, al toser, cubrirse la boca y la nariz con el codo flexionado o con un pañuelo. Estas pueden ser ajustadas de acuerdo a las condiciones sanitarias de su localidad y su lugar de trabajo.

En cuanto al Perú, es oportuno señalar que el domingo 15 de marzo de 2020 se emitió un D. S. Nº 044-2020PCM, declarando el Estado de Emergencia Nacional por las graves circunstancias que afectan la vida de la Nación a consecuencia del brote del COVID-19 y se establecieron las siguientes medidas: suspensión del ejercicio de Derechos Constitucionales, limitación al ejercicio del derecho a la libertad de tránsito de las personas, restricciones en el ámbito de la actividad comercial, actividades culturales, establecimientos y actividades recreativas, hoteles y restaurantes, cierre temporal de fronteras, reducción de la oferta de operaciones en cincuenta por ciento $(50 \%)$ en el territorio nacional por medio terrestre y fluvial, intervención de la Policía Nacional del Perú y de las Fuerzas Armadas, a fin de garantizar la implementación.

Estas medidas de carácter drástico para el momento en que fueron implementadas, se han ido flexibilizando progresivamente motivado a que se observa que las consecuencias de la pandemia COVID 19 han disminuido.

\section{Metodología para la valoración de impactos ambientales \\ Criterios a evaluar}

Existen numerosos modelos y procedimientos para la evaluación de impactos sobre el ambiente o sobre alguno de sus factores; algunos generales, con pretensiones de universalidad, otros específicos para situaciones o aspectos concretos; algunos cualitativos, otros operando con amplias bases de datos e instrumentos de cálculo sofisticados; de carácter estático unos, dinámico otros, etc. Para la presente nota se utilizan algunos criterios tomados de la metodología expuesta por Conesa (1997) (Tabla 1).

Puntaje por criterio

A objeto de obtener una puntuación máxima de 100 
Tabla 1. Criterios a evaluar.

\begin{tabular}{|c|c|c|c|}
\hline Criterios & & Significado & Rango \\
\hline Naturaleza & $\mathrm{N}$ & $\begin{array}{c}\text { Hace alusión al carácter benéfico }(+) 10 \text { o perjudicial }(-) 2,5 \text {, de } \\
\text { las distintas acciones que van a actuar sobre las distintas } \\
\text { medidas implementadas. }\end{array}$ & $\begin{array}{c}\text { Negativo } 2,5 \\
\text { Positivo } 10\end{array}$ \\
\hline Intensidad & IN & $\begin{array}{c}\text { Grado de incidencia de la acción sobre el factor en el ámbito } \\
\text { específico en el que actúa. Varía entre } 2,5 \text { y } 30 \text {, siendo } 30 \text { la } \\
\text { expresión total del factor en el área en la que se produce el } \\
\text { efecto y } 2,5 \text { una mínima. }\end{array}$ & $\begin{array}{l}\text { Baja } 2,5 \\
\text { Media } 16,25 \\
\text { Total } 30\end{array}$ \\
\hline Extensión & EX & $\begin{array}{l}\text { Área de influencia teórica del impacto en relación con el } \\
\text { entorno de la actividad (\% de área, respecto al entorno, en que } \\
\text { se manifiesta el efecto). Si la acción produce un efecto muy } \\
\text { localizado, se considera que el impacto tiene un carácter } \\
\text { puntual }(2,5) \text {. Si, por el contrario, el impacto no admite una } \\
\text { ubicación precisa del entorno de la actividad, teniendo una } \\
\text { influencia generalizada en todo él, el impacto será Crítico (30). }\end{array}$ & $\begin{array}{l}\text { Puntual } 2,5 \\
\text { Parcial } 5 \\
\text { Extenso } 10 \\
\text { Total } 20 \\
\text { Critica } 30\end{array}$ \\
\hline Momento & $\mathrm{MO}$ & $\begin{array}{l}\text { Alude al tiempo entre la aparición de la acción que produce el } \\
\text { impacto y el comienzo del efecto sobre el factor considerado } \\
\text { (recursos hídricos). Si el tiempo transcurrido es nulo, el } \\
\text { momento será Inmediato, y si es inferior a un año, Corto plazo, } \\
\text { asignándole en ambos casos un valor de (20). Si es un período } \\
\text { de tiempo mayor a cinco años, Largo Plazo }(2,5) \text {. }\end{array}$ & $\begin{array}{l}\text { Largo plazo } 2,5 \\
\text { Mediano plazo } 5 \\
\text { Inmediato } 10 \\
\text { Critico } 20\end{array}$ \\
\hline Sinergia & SI & $\begin{array}{l}\text { Este atributo contempla el reforzamiento de dos o más efectos } \\
\text { simples. La componente total de la manifestación de los efectos } \\
\text { simples, provocados por acciones que actúan simultáneamente, } \\
\text { es superior a la que cabría de esperar cuando las acciones que } \\
\text { las provocan actúan de manera independiente, no simultánea. }\end{array}$ & $\begin{array}{l}\text { Sin sinergismo } 2,5 \\
\text { Sinérgico } 5 \\
\text { Muy sinérgico } 10\end{array}$ \\
\hline
\end{tabular}

puntos cada medida implementada y tomando como referencia los puntajes asignados por Conesa (1997) para cada criterio, se procedió a realizar un reparto proporcional en función de los cinco criterios seleccionados, estableciéndose los puntajes mínimos y máximos (Tabla 2).

\section{Condición resultante}

El resultado total de la aplicación de los criterios para

Tabla 2. Puntaje por criterio

\begin{tabular}{ccccc}
\hline \multirow{2}{*}{ Criterios } & \multicolumn{2}{c}{$\begin{array}{c}\text { Puntaje (Conesa, } \\
\text { 1997) }\end{array}$} & \multicolumn{2}{c}{$\begin{array}{c}\text { Puntaje } \\
\text { (Propuesto) }\end{array}$} \\
\cline { 2 - 5 } & Mínimo & Máximo & Mínimo & Máximo \\
\hline Naturaleza & 1 & 4 & 2,5 & 10 \\
Intensidad & 1 & 12 & 2,5 & 30 \\
Extensión & 1 & 12 & 2,5 & 30 \\
Momento & 1 & 8 & 2,5 & 20 \\
Sinergia & 1 & 4 & 2,5 & 10 \\
Total & 5 & 40 & 7,5 & 100 \\
\hline
\end{tabular}

cada una de las medidas implementadas, generará un valor que oscilará entre 62,500 y 500,000, conformándose cuatro categorías o condiciones que responden a las medidas de posición de la distribución de los datos, específicamente, a lo cuartiles (Tabla 3 ).

\section{RESULTADOS}

Una vez asignados los puntajes a cada uno de los criterios para el estado actual de los recursos hídricos, encontramos que las valoraciones indican que en la medida 1 (Suspensión del ejercicio de Derechos Constitucionales) el menor valor resulta del criterio (momento) y el mayor valor del criterio (intensidad), así sucesivamente hasta la medida 5 (llevar mascarilla) (Tabla 4).

Con respecto a la medida implementada "Suspensión del ejercicio de Derechos Constitucionales", se observa que el criterio momento obtuvo la valoración más baja (10) y el criterio extensión el valor más alto (20). En cuanto a la medida implementada "Limitación al ejercicio del derecho a la libertad de tránsito de las personas", el criterio que obtuvo la valoración baja es extensión (20) y los demás criterios obtuvieron la valoración máxima (10, 20,30y 10).

En referencia a la medida implementada "Restricciones en el ámbito de la actividad comercial, actividades culturales, establecimientos y actividades recreativas, 
Tabla 3. Condición resultante

\begin{tabular}{cccc}
\hline \multirow{2}{*}{ Condición } & \multicolumn{2}{c}{ Valor } & Observación \\
\cline { 2 - 3 } & Mínimo & Máximo & Muy baja incidencia en los recursos hídricos. \\
Muy baja & 62,500 & 171,875 & \\
Baja & 171,875 & 281,250 & Baja incidencia en los recursos hídricos. \\
Moderada & 281,250 & 390,625 & \\
Alta & 390,625 & 500,000 & Moderada incidencia en los recursos hídricos. \\
\hline
\end{tabular}

Tabla 4. Impactos de las medias implementadas durante la pandemia COVID 19 en el estado de los recursos hídricos.

\begin{tabular}{|c|c|c|c|}
\hline Medidas implementadas & Criterios & $\begin{array}{l}\text { Descripción } \\
\text { (valoración) }\end{array}$ & Sub total \\
\hline \multirow{5}{*}{$\begin{array}{l}\text { Suspensión del ejercicio de } \\
\text { Derechos Constitucionales }\end{array}$} & Naturaleza & Positivo: 10 & \multirow{5}{*}{66,25} \\
\hline & Intensidad & Media: 16,25 & \\
\hline & Extensión & Total: 20 & \\
\hline & Momento & Inmediato: 10 & \\
\hline & Sinergia & Muy sinérgico: 10 & \\
\hline \multirow{5}{*}{$\begin{array}{c}\text { Limitación al ejercicio del } \\
\text { derecho a la libertad de tránsito } \\
\text { de las personas }\end{array}$} & Naturaleza & Positivo: 10 & \multirow{5}{*}{90} \\
\hline & Intensidad & Total: 30 & \\
\hline & Extensión & Total: 20 & \\
\hline & Momento & Crítico: 20 & \\
\hline & Sinergia & Muy sinérgico: 10 & \\
\hline \multirow{5}{*}{$\begin{array}{l}\text { Restricciones en el ámbito de la } \\
\text { actividad comercial, actividades } \\
\text { culturales, establecimientos y } \\
\text { actividades recreativas, hoteles } \\
\text { y restaurantes. }\end{array}$} & Naturaleza & Positivo: 10 & \multirow{5}{*}{61,25} \\
\hline & Intensidad & Media: 16,25 & \\
\hline & Extensión & Total: 20 & \\
\hline & Momento & Inmediato: 10 & \\
\hline & Sinergia & Sinérgico: 5 & \\
\hline \multirow{5}{*}{ Cierre temporal de fronteras } & Naturaleza & Positivo: 10 & \multirow{5}{*}{100} \\
\hline & Intensidad & Total: 30 & \\
\hline & Extensión & Crítico: 30 & \\
\hline & Momento & Crítico: 20 & \\
\hline & Sinergia & Muy sinérgico:10 & \\
\hline \multirow{6}{*}{ Llevar mascarilla } & Naturaleza & Negativo: 2,5 & \multirow{5}{*}{38,75} \\
\hline & Intensidad & Media: 16,25 & \\
\hline & Extensión & Extenso: 10 & \\
\hline & Momento & Mediano plazo: 5 & \\
\hline & Sinergia & Sinérgico: 5 & \\
\hline & Total & & 356,25 \\
\hline
\end{tabular}

hoteles y restaurantes", los criterios que obtuvieron valoración baja es momento (10) y sinergia (5), mientras que criterio con valoración más alta es extensión (20).

En el caso del resultado de la medida implementada "Cierre temporal de fronteras", se debe indicar que todos los criterios obtuvieron la misma puntuación, es decir, el valor máximo permisible (10,30, 30, 20 y 10). En la medida implementada "Llevar mascarilla" se obtuvo la puntuación más baja en el criterio naturaleza $(2,5)$, es decir un impacto negativo y en el criterio extensión (10) la puntuación más alta.

Es preciso señalar que la medida que obtuvo menor puntaje es "Llevar mascarilla" y la que obtuvo mayor puntaje es "Cierre temporal de fronteras".

\section{CONCLUSIONES}

Una vez evaluados los criterios naturaleza, intensidad, extensión, momento y sinergia para cada una de las cinco medidas implementadas, se observa que la medida con mayor puntaje es el cierre temporal de fronteras con 100, y la medida con menor puntaje es llevar mascarilla con 38,75 ; mientras que el puntaje total es 356,25 , que se traduce en una moderada incidencia en los recursos hídricos. 


\section{CONTRIBUCIÓN DE LOS AUTORES}

Milla Pino Manuel Emilio, fue el encargado de la redacción del documento y adecuación a las normas de la revista; Torres Cruz María Marleni fue la responsable de la recopilación de información referida al tema en estudio; y Rojas Milla Cristian Jesús realizó la sistematización de la información.

\section{CONFLICTO DE INTERESES}

Los autores declaran no tener conflicto de intereses

\section{REFERENCIAS BIBLIOGRÁFICAS}

AQUASTAT. 2021. AQUASTAT - FAO's Global Information System on Water and Agriculture. http://www.fao.org/aquastat/en/. (Consultada el 20 de marzo de 2021)

ANA (Autoridad Nacional del Agua). 2016. Estrategia Nacional Para El Mejoramiento De La Calidad De Los Recursos Hídricos. Lima (Perú): MINAGRI.

Burek, P., Y. Satoh, G. Fischer, M. T. Kahil, A. Scherzer, S. Tramberend, L. F. Nava, Y. Wada, S. Eisner, M. Flörke, N. Hanasaki, P. Magnuszewski, B. Cosgrove, y D. Wiberg. 2016. Water Futures and Solution. Reporte Final. Laxenburg (Austria).

Conesa, V. 1997. Auditorías medioambientales: Guía metodológica. Madrid (España): MundiPresna.

Hunter, P. R., A. M. MacDonald, y R. C. Carter. 2010. "Water Supply and Health". PLoS Med 7 (11): e $\begin{array}{llllllllll}1 & 0 & 0 & 0 & 3 & 6 & 1\end{array}$. D O I : 10.1371/journal.pmed.100036.

Ki-moon, B. 2014. Departamento de Asuntos Económicos y Sociales de Naciones Unidas. https://www.un.org/spanish/waterforlifedeca de/quality.shtml. (Consultada el 20 de marzo de 2021)

Wada, Y., M. Flörke, N. Hanasaki, S. Eisner, G. Fischer, S. Tramberend, Y. Satoh, M. T. H. Van Vliet, P. Yillia, C. Ringler, P. Burek, y D. Wiberg. 2016. "Modelling global water use for the 21st century: The Water Futures and Solutions (WFaS) initiative and itsapproaches". Geosci. Model Dev. 9: 175222. DOI: $10.5194 /$ gmd-9-175-2016.

WIDENZA CONSULTORES. 2021. Propuestas del Bicentenario. Análisis y evidencias para la

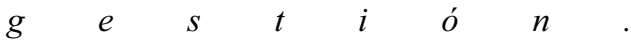
https://propuestasdelbicentenario.pe/wpcontent/uploads/2021/02/Recursoshidricos.pdf. (Consultada el 19 de marzo de 2021)

WWAP (Programa Mundial de Evaluación de los Recursos Hídricos de la UNESCO). 2018. Informe Mundial de las Naciones Unidas sobre el Desarrollo de los Recursos Hidricos 2018: Soluciones basadas en la naturaleza para la gestión del agua. París (Francia): UNESCO.

WWAP (Programa Mundial de Evaluación de los Recursos Hídricos de la UNESCO). 2019. Informe Mundial de las Naciones Unidas sobre el Desarrollo de los Recursos Hidricos 2019: No dejar a nadie atrás. París (Francia): UNESCO.

WWAP (Programa Mundial de Evaluación de los Recursos Hídricos de la UNESCO). 2020. Informe Mundial de las Naciones Unidas sobre el Desarrollo de los Recursos Hídrico 2020: Agua y Cambio Climático. París (Francia): UNESCO.

WWAP (Programa Mundial de Evaluación de los Recursos Hídricos de la UNESCO). 2021. Valuing Water. París (Francia): UNESCO. 